\title{
A PORTABLE ELECTRIC BASS USING TWO PDAS
}

\author{
*Tsutomu TERADA, **Masahiko TSUKAMOTO, **Shojiro NISHIO \\ * Cybercommunity Division, Cybermedia Center, Osaka University \\ 5-1 Mihogaoka, Ibaraki, Osaka 567-0047, JAPAN \\ +81-6-6879-8796 \\ tsutomu@cmc.osaka-u.ac.jp \\ ** Dept. of Information Systems Engineering, Grad. School of Engineering, Osaka University \\ 2-1 Yamadaoka, Suita, Osaka 565-0871, JAPAN \\ +81-6-6879-7821 \\ \{tuka,nishio\}@ise.eng.osaka-u.ac.jp
}

\begin{abstract}
The DoublePad/Bass is a musical instrument that consists of two PDAs with touch panel displays. A user holds a PDA in each hand as if playing an electric bass. The left hand specifies a note on one PDA and the right hand touches the touch panel of the other PDA to generate a sound. Musicians who can play an electric bass should be easily able to play the DoublePad/Bass.
\end{abstract}

Key words: $\quad$ Musical Instruments, Electric Bass, PDA

\section{Introduction}

Advanced mobile computing technologies have made it easy for people to carry PDAs (personal digital assistants) with them wherever they go. PDAs are used for various purposes, and we expect them to become an increasingly important source of entertainment, especially for music ${ }^{[2]}$. However, while several applications allow users to listen to music, few enable users to actually perform music or collaborate with other musicians. Therefore, the goal of our study has been to develop the DoublePad/Bass, a mobile electronic musical instrument that enables users to perform music anywhere. The DoublePad/Bass uses two PDAs with touch panel displays.

The original version of this chapter was revised: The copyright line was incorrect. This has been corrected. The Erratum to this chapter is available at DOI: 10.1007/978-0-387-35660-0_65 
One PDA is used for the input of each hand with the input method being similar to that of a real electric bass.

The remainder of this paper is organized as follows. Section 2 outlines the features of the DoublePad/Bass, Section 3 explains the system design, and Section 4 discusses the implementation of a prototype system and our plans for future modifications. We conclude in Section 5.

\section{Doublepad/Bass}

Most musical instruments are played using both hands. Therefore, their operation can be simulated by using a PDA in each hand with similar control operations being realized through touch panel displays. We call two PDAs being used in this way the DoublePad. In this research, we applied this framework to design the DoublePad/Bass which can be used as an electric bass. Other musical instruments, such as the guitar, piano, maraca, violin, samisen, and accordion should also be possible to realize through this framework.

The DoublePad/Bass has the following features:

\section{No special equipment required}

The inconvenience of carrying a bulky musical instrument is avoided.

Many people normally carry a PDA that (with a partner) can become a musical instrument. Therefore, a user can enjoy playing music anytime and anywhere, such as while on a stroll or commuting. In addition, they can easily engage in impromptu jam sessions with anybody they meet.

\section{Visual recognition of a player's performance}

Many musicians enjoy demonstrating their technique to audiences while playing. With the DoublePad/Bass, the player's technical skill can be visually appreciated because the player's actions will be similar to those when playing an actual instrument.

\section{Simulation of a normal bass in play}

When musicians play an actual electric bass, he uses various techniques such as hammering-on, pulling-off, slide, and choking. On the DoublePad/Bass, the same techniques can be reproduced by performing similar operations on a PDA. Accordingly, a player who has mastered these techniques on an actual electric bass can use similar techniques on the DoublePad/Bass. Conversely, techniques learned on the DoublePad/Bass can be applied on actual musical instruments. 


\section{Design of the Doublepad/Bass}

\subsection{Input methods}

Two PDAs, each with a touch panel display, are held by the user - one in each hand. The PDA screens display the strings and frets of an actual electric bass (Fig. 1). Four horizontal lines on the screen for the right hand represent strings, and a player makes a sound by passing his finger over one of the lines (Fig. 2). In the screen for the left hand (Fig. 3), horizontal lines again represent strings and vertical lines represent the frets used to control the pitch. A player determines the pitch by pressing on a string at a fret as with an actual electric bass.

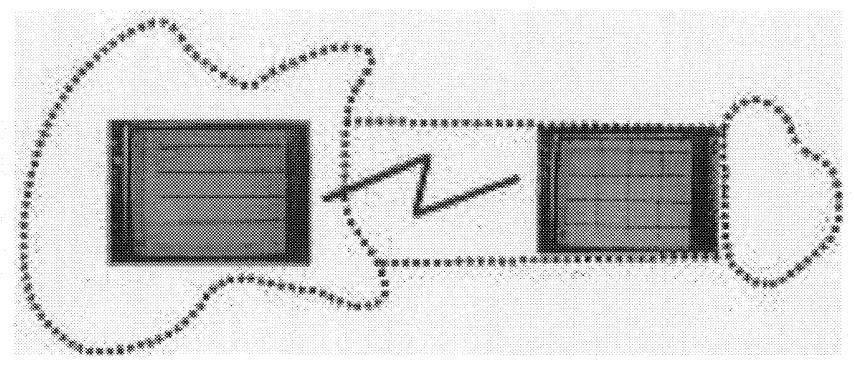

Figure 1. Basic concept of the DoublePad/Bass
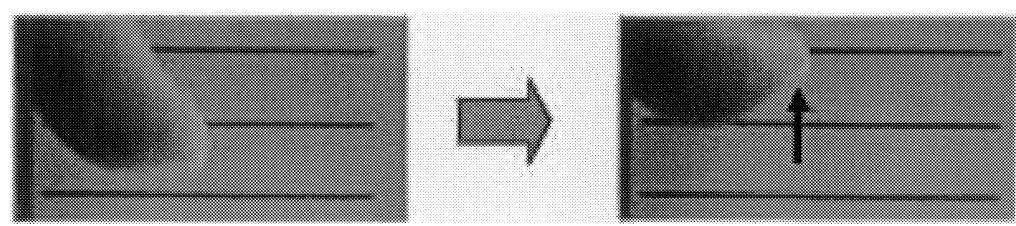

Figure 2. Operation of the right hand
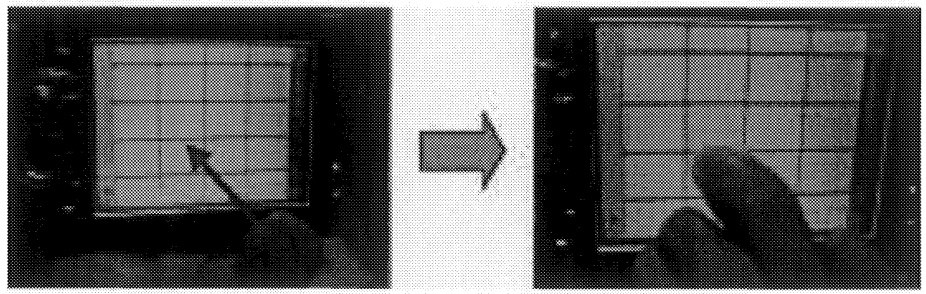

Figure 3. Operation of the left hand 


\subsection{Realizing dual-touch operation}

Although a touch panel display on a PDA normally allows only one input at a time, two or more fingers often simultaneously press strings when an actual electric bass is being played. Therefore, we used the Dual Touch technique which enables the detection of simultaneous-input at two points in the DoublePad/Bass ${ }^{[1]}$. The Dual Touch technique processes two simultaneous inputs on a PDA by calculating a center point between the two input points. This enables players to attain a musical performance close to that of an actual musical instrument. Musicians capable of playing techniques mastered on an actual musical instrument can achieve a more advanced performance through this function. Likewise, the techniques acquired with this system can be applied to an actual musical instrument.

\subsection{Reflecting playing techniques}

When a musician plays an actual electric bass, the left hand is frequently used for certain playing techniques. Here, we explain how the DoublePad/Bass allows use of some of these techniques.

- Hammering-on: a technique to change the sound pitch without a righthand operation by throwing a finger into the area of higher pitch when a sound is reading out.

- Pulling-off: a technique to change the sound pitch without a right-hand operation by lifting one finger with sufficient vigor when two fingers are pressing down.

- Slide: a technique to change the sound pitch by moving a finger on the left hand while it presses down on a string.

- Choking: a technique to change the sound pitch by shifting to a higher or lower string where a finger presses down in the same position.

The left-hand input for these techniques is shown in Figs. 4 to 7. Some of the techniques need simultaneous input at two or more points on a PDA, which is enabled by the Dual Touch technique. 

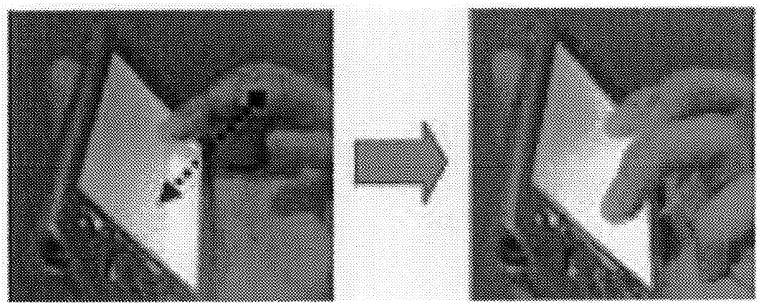

Figure 4. Hammering-on
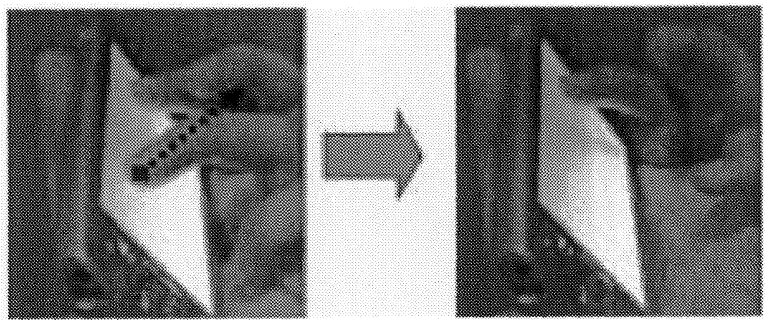

Figure 5. Pulling-off
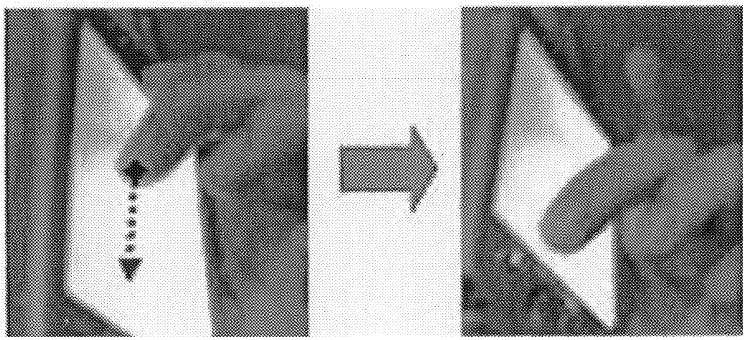

Figure 6. Slide
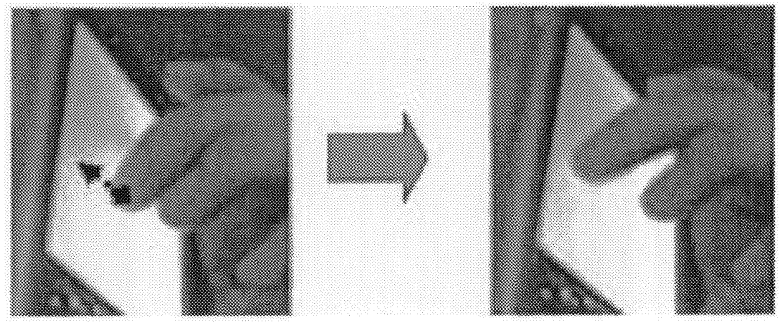

Figure 7. Choking 


\section{Implementation}

We implemented a prototype system of the DoublePad/Bass (Fig. 8), and a user playing music with the system is shown in Fig. 9. Each PDA was a Pocket PC, and we developed the system using eMbedded Visual Tools 3.0.

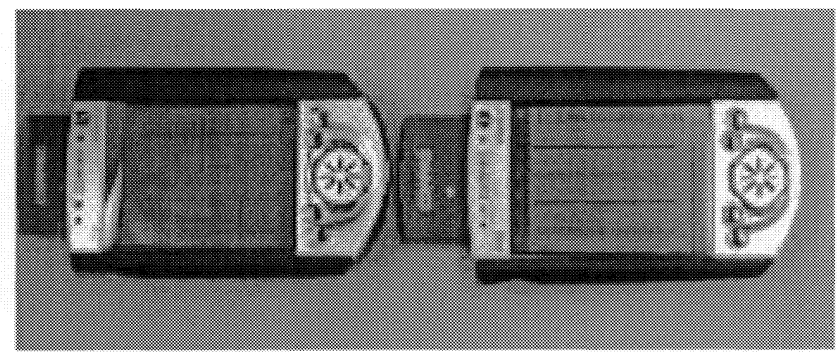

Figure 8. A prototype system

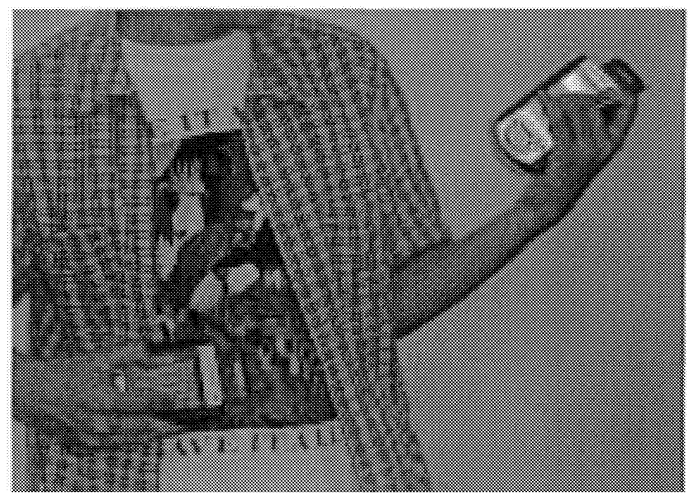

Figure 9. Playing music with the DoublePad/Bass

\subsection{Communication method}

In the DoublePad/Bass, the operations of the two hands obviously have to be coordinated, and there are two alternative forms of communication that can be used to achieve this. In one, the left-hand device continuously sends information to the right-hand device, and the right-hand device emits sound when a finger 'plucks' a string. In the other, the right-hand device transmits information to the left-hand device when a string is played on the right-hand device, and the left-hand device emits a sound by integrating the received information and actual state of the left-hand device. 
Although the system emits a sound with almost no delay from the righthand input when the first method is used, considerable data traffic can arise from the information being continuously sent to the right-hand device from the left-hand device. If this traffic causes a communication delay, the sound may be created with a different pitch than the player intended.

When the second method is used, the system can instantaneously react to the left-hand operations (slide, hammering-on, etc.), but any communication delay will distort the sound generation of the right hand.

Which response is more important, that to the right-hand sound generation or that to the left-hand operations, will depend on the situation and the user's intent. Therefore, the communication method in the DoublePad/Bass can be switched according to the user's preference.

\subsection{Sound source}

We considered three possible output sound sources: WAVE sounds, software MIDI sounds, and external MIDI sounds. External MIDI sounds would make playing techniques easy to reproduce since the pitch and tone can be freely adjusted. With WAVE sounds, a music scale is expressed by preparing sampling sounds. However, MIDI sounds cannot be used in the present Pocket PC, so we implemented a DoublePad/Bass with only WAVE sounds as the prototype system. Although users cannot smoothly change the pitch in this prototype, sound can be output without external equipment. Implementation of a system using MIDI sounds is planned as future work.

\subsection{Fret transposition and the screen layout}

With an actual electric bass, the left hand covers over 20 frets. However, only limited information can be displayed on a PDA screen at any one time, so we had to change the information display in some way. Although the displayed information can be changed by pressing a button in this prototype, this does not allow the audience to see the position at which the player presses down to obtain a certain pitch. We plan to develop an improved method for changing information on the screen according to the user's action in our future work. We are considering two methods:

- When a user moves his left hand, frets on the screen can also be made to move automatically by using supplementary equipment such as a curtain rail. However, while this would have the benefit of making the hand position visually apparent, supplementary equipment would make the system less portable and convenient to use. 
- The system can determine the position of the left hand by using a device that detects the distance between the left-hand and right-hand devices. This method would allow visual expression of the hand position without making the system more awkward to use, but would require a device that can detect the relative positions extremely accurately.

In this prototype system, four frets were displayed on the screen at a time. In our future work, we also plan to work on the regulation of a moderate interval between frets and a means to change the fret intervals according to the situation.

\section{Conclusion}

The DoublePad/Bass is a portable musical instrument that consists of two PDAs. It enables a playing style and performance similar to those of an actual electric bass by assigning a PDA to each hand. This system allows users to perform music and collaborate with other musicians anywhere at anytime.

\section{References}

[1] Matsushita, N., Ayatsuka, Y., Rekimoto, J., "Dual Touch: a New Interaction Technique for Pen-Based PDAs," in Proc. of Workshop on Interactive Systems and Software (WISS'99), pp. 23-32 (Dec. 1999).

[2] Nishimoto, K., Maekawa, T., Tada, Y., Mase, K., Nakatsu, R., "Networked Wearable Musical Instruments Will Bring a New Musical Culture" in Proc. of the Fifth International Symposium on Wearable Computers (ISWC2001), pp. 55-62 (Oct. 2001). 